\title{
On the Norms of $r$-Hankel Matrices Involving Fibonacci and Lucas Numbers
}

\author{
Hasan Gökbaş ${ }^{1 *}$, Hasan Köse ${ }^{2}$ \\ ${ }^{1}$ Şemsi Tebrizi Girl Anatolian Religious Vocational High School, Konya, Turkey \\ ${ }^{2}$ Science Faculty, Selçuk Universty, Konya, Turkey \\ Email: ^hgokbas@hotmail.com, hkose@selcuk.edu.tr
}

How to cite this paper: Gökbaş, H. and Köse, H. (2018) On the Norms of r-Hankel Matrices Involving Fibonacci and Lucas Numbers. Journal of Applied Mathematics and Physics, 6, 1409-1417.

https://doi.org/10.4236/jamp.2018.67117

Received: May 15, 2018

Accepted: July 7, 2018

Published: July 10, 2018

Copyright $\odot 2018$ by authors and Scientific Research Publishing Inc. This work is licensed under the Creative Commons Attribution International License (CC BY 4.0).

http://creativecommons.org/licenses/by/4.0/

\begin{abstract}
Let us define $A=H_{r}=\left(a_{i j}\right)$ to be $n \times n \quad r$-Hankel matrix. The entries of matrix $A$ are $F_{n}=F_{i+j-2}$ or $L_{n}=F_{i+j-2}$ where $F_{n}$ and $L_{n}$ denote the usual Fibonacci and Lucas numbers, respectively. Then, we obtained upper and lower bounds for the spectral norm of matrix $A$. We compared our bounds with exact value of matrix $A$ 's spectral norm. These kinds of matrices have connections with signal and image processing, time series analysis and many other problems.
\end{abstract}

\section{Keywords}

Euclidean Norm, Spectral Norm, $r$-Hankel Matrix, Fibonacci Numbers, Lucas Numbers

\section{Introduction}

"Special matrices" is a widely studied subject in the research area of matrix analysis. Especially, special matrices whose entries are well known number sequences have become a very interesting research subject in recent years and many authors have obtained some good results in this area. Some researches denoted the norms of the special matrices involving famous number sequences. The authors found lower bounds, upper bounds and exact values for the spectral norms of these matrices.

Our aim in this study is to obtain some norm bounds more closure than those in literature to the exact value of matrix $A$ 's spectral norm. Lots of articles which concern estimates for spectral norms of special matrices have been written so far. 
Solak has studied the norms of circulant matrices with fibonacci and lucas numbers in [1], Türkmen and Gökbaş have made a similar study by using the r-circulant matrix with pell and pell-lucas numbers in [2], Shen and Cen have made a similar study by using the same special matrix with k-fibonacci and k-lucas numbers in [3], Akbulak and Bozkurt found lower and upper bounds for the spectral norms of toeplitz matrices with classical fibonacci and lucas numbers entries in [4], Shen gave upper and lower bounds for the spectral norms of toeplitz matrices with k-fibonacci and k-lucas numbers entries in [5], Akbulak and Bozkurt have made a similar study by using the hankel matrix with fibonacci and lucas numbers in [6], Gökbaş and Türkmen gave upper and lower bounds for the spectral norms of $r$-toeplitz matrices involving fibonacci and lucas numbers in [7], Bozkurt and Tam obtained determinants and inverse of circulant matrices with jacobsthal and jacobsthal-lucas numbers in [8].

The Fibonacci and Lucas sequences $F_{n}$ and $L_{n}$ are defined by the recurrence relations (Table 1 )

$$
\begin{array}{ll}
F_{0}=0, F_{1}=1, F_{n}=F_{n-1}+F_{n-2} \text { for } n \geq 2 \\
L_{0}=2, L_{1}=1, L_{n}=L_{n-1}+L_{n-2} \text { for } n \geq 2
\end{array}
$$

The following sum formulas the Fibonacci and Lucas numbers are well known [9]:

$$
\begin{gathered}
\sum_{k=1}^{n-1} F_{k}^{2}=F_{n} F_{n-1} \\
\sum_{k=1}^{n-1} L_{k}^{2}=L_{n} L_{n-1}-2 \\
\sum_{k=1}^{n} k F_{k}^{2}= \begin{cases}n F_{n} F_{n+1}-F_{n}^{2}+1, & \text { if } n \text { is odd } \\
n F_{n} F_{n+1}-F_{n}^{2}, & \text { otherwise }\end{cases} \\
\sum_{k=1}^{n} k L_{k}^{2}= \begin{cases}n L_{n} L_{n+1}-L_{n}^{2}-1, & \text { if } n \text { is odd } \\
n L_{n} L_{n+1}-F_{n}^{2}+4, & \text { otherwise }\end{cases}
\end{gathered}
$$

The Euclidean norm of the matrix $A$ is defined as

$$
\|A\|_{E}=\left(\sum_{i, j=1}^{n}\left|a_{i j}\right|^{2}\right)^{1 / 2}
$$

The singular values of the matrix $A$ is

$$
\sigma_{i}=\sqrt{\lambda_{i}\left(A^{*} A\right)}
$$

where $\lambda_{i}$ is an eigenvalues of matrix $A^{*} A$ and $A^{*}$ is conjugate transpose of the matrix $A$. The square roots of the maximum eigenvalues of $A^{*} A$ are called the spectral norm of $A$ and are induced by $\|A\|_{2}$.

Table 1. The Fibonacci and Lucas sequence have been given.

\begin{tabular}{cccccccccccccc}
\hline$n$ & 0 & 1 & 2 & 3 & 4 & 5 & 6 & 7 & 8 & 9 & 10 & 11 & $\ldots$ \\
$F_{n}$ & 0 & 1 & 1 & 2 & 3 & 5 & 8 & 13 & 21 & 34 & 55 & 89 & $\ldots$ \\
$Q_{n}$ & 2 & 1 & 3 & 4 & 7 & 11 & 18 & 29 & 47 & 76 & 123 & 199 & $\ldots$ \\
\hline
\end{tabular}


The following imequality holds,

$$
\frac{1}{\sqrt{n}}\|A\|_{E} \leq\|A\|_{2} \leq\|A\|_{E}
$$

Define the maximum row length norm $r_{1}$ and the maximum column length norm $c_{1}$ of any matrix $A$ by

$$
r_{1}(A)=\max _{i} \sqrt{\sum_{j}\left|a_{i j}\right|^{2}} \text { and } c_{1}(A)=\max _{j} \sqrt{\sum_{i}\left|a_{i j}\right|^{2}}
$$

respectively. Let $A, B$ and $C$ be $m \times n$ matrices. If $A=B \circ C$ then

$$
\|A\|_{2} \leq r_{1}(B) c_{1}(C)
$$

for the matrices $A=\left(a_{i j}\right)_{m \times n}$ and $B=\left(b_{i j}\right)_{m \times n}$ the Hadamard Product of these matrices is defined as $A \circ B=\left(a_{i j} b_{i j}\right)_{m \times n}[10]$.

Let us give some lemmas which we will use in our result.

Lemma 1.1: Be a Hankel matrix whose entries determined by $a_{i j}=F_{i+j-2}$ where $F_{n}$ stands for $n$th Fibonacci numbers [6].

$$
\begin{gathered}
\sqrt{-\frac{F_{4 n}-3 F_{2 n}^{2}-2 F_{2 n}+6 F_{n}^{2}-2+2[-1]^{n}}{2 n}} \leq\|A\|_{2} \\
\|A\|_{2} \leq \sqrt{\left(F_{2 n-2} F_{2 n-3}-F_{n-1} F_{n-2}+F_{2 n-2}^{2}\right)\left(F_{2 n-2} F_{2 n-3}-F_{n-1} F_{n-2}+1\right)}
\end{gathered}
$$

Lemma 1.2: Be a Hankel matrix whose entries determined by $a_{i j}=F_{i+j-2}$ where $F_{n}$ stands for $n$th Fibonacci numbers [11].

$$
\|A\|_{2}= \begin{cases}\frac{F_{2 n-1}-1+\sqrt{F_{2 n-1}^{2}-2 F_{n-1}+4 F_{n}^{2}-3}}{2}, & \text { if } n \text { is odd } \\ \frac{F_{2 n-1}-1+\sqrt{F_{2 n-1}^{2}-2 F_{n-1}+4 F_{n}^{2}+1}}{2}, & \text { otherwise }\end{cases}
$$

Lemma 1.3: Be a Hankel matrix whose entries determined by $a_{i j}=L_{i+j-2}$ where $L_{n}$ stands for $n$th Lucas numbers [6].

$$
\sqrt{\frac{L_{2 n-1}^{2}-2 L_{n-1}^{2}+1+5\left[1-(-1)^{n}\right]}{n}} \leq\|A\|_{2}
$$

Lemma 1.4: Be a Hankel matrix whose entries determined by $a_{i j}=L_{i+j-2}$ where $L_{n}$ stands for $n$th Lucas numbers [11].

$$
\|A\|_{2}= \begin{cases}\frac{L_{2 n-1}+1+\sqrt{5\left(\frac{L_{2 n-2}+L_{2 n}-5}{5}\right)^{2}+4}}{2}, & \text { if } n \text { is odd } \\ \frac{L_{2 n-1}+1+\sqrt{5\left(\frac{L_{2 n-2}+L_{2 n}-5}{5}\right)^{2}}}{2}, & \text { otherwise }\end{cases}
$$




\section{Result}

In this section, we define an $n \times n \quad r$-Hankel matrix $A=H_{r}=\left(a_{i j}\right)$ whose entries are $a_{i j}=F_{i+j-2}$ or $a_{i j}=L_{i+j-2}$ where $F_{n}$ and $L_{n}$ denote the usual Fibonacci and Lucas numbers, respectively. We obtain some inequalities related to $A=H_{r}=\left(a_{i j}\right)$.

Definition 2.5: $A$ matrix $H_{r}=\left(h_{i j}\right) \in M_{n, n}(\mathcal{C})$ is called a $r$-Hankel matrix if it is of the form

$$
H_{i j}= \begin{cases}r h_{i+j-2}, & i+j \leq n \\ h_{i+j-2}, & i+j>n\end{cases}
$$

Obviously, the $r$-Hankel matrix $H$ is determined by parameter $r$ and its first row elements $h_{0}, h_{1}, \cdots, h_{n-1}$, thus we denote $H=H_{r}\left(h_{0}, h_{1}, \cdots, h_{n-1}\right)$. For $r=1$, the matrix $H$ is called a Hankel matrix.

Theorem 2.6: Let $A=H_{r}=\left(a_{i j}\right)$ be a Hankel matrix satisfying $a_{i j}=F_{i+j-2}$, where $r \in(\mathcal{C})$.

1) $|r| \geq 1, \sqrt{\frac{F_{2 n-1} F_{2 n-2}+F_{2 n-2}^{2}-3 F_{n-1} F_{n-2}-2 F_{n-2}^{2}+F_{n} F_{n-1}+\left[1-(-1)^{n}\right]}{n}} \leq A_{2}$

$$
\|A\|_{2} \leq \sqrt{\left(|r|^{2}(n-1)+1\right)\left(F_{2 n-1} F_{2 n-2}-F_{n-1} F_{n-2}\right)}
$$

2) $|r|<1,|r| \sqrt{\frac{F_{2 n-1} F_{2 n-2}+F_{2 n-2}^{2}-3 F_{n-1} F_{n-2}-2 F_{n-2}^{2}+F_{n} F_{n-1}+\left[1-(-1)^{n}\right]}{n}} \leq\|A\|_{2}$

$$
\|A\|_{2} \leq \sqrt{n\left(F_{2 n-1} F_{2 n-2}-F_{n-1} F_{n-2}\right)}
$$

where $\|\cdot\|_{2}$ is the spectral norm and $F_{n}$ denotes the $n$th Fibonacci number.

Proof. The matrix $A$ is of the form

$$
A=\left[\begin{array}{cccc}
r F_{0} & r F_{1} & \cdots & F_{n-1} \\
r F_{1} & & \cdots & F_{n} \\
\vdots & \vdots & \ddots & \vdots \\
F_{n-1} & F_{n} & \cdots & F_{2 n-2}
\end{array}\right]
$$

Then,

$$
\|A\|_{F}^{2}=\sum_{k=0}^{n-2}|r|^{2}(k+1) F_{k}^{2}+\sum_{k=n-1}^{2 n-2}(2 n-1-k) F_{k}^{2} .
$$

Hence, when $|r| \geq 1$ we obtain

$$
\begin{aligned}
\|A\|_{F}^{2} & \geq \sum_{k=0}^{n-2}(k+1) F_{k}^{2}+\sum_{k=n-1}^{2 n-2}(2 n-1-k) F_{k}^{2} \\
& \geq(2 n-1) \sum_{k=0}^{n-2} F_{k}^{2}-\sum_{k=1}^{2 n-2} k F_{k}^{2}-(2 n-1) \sum_{k=0}^{n-2} F_{k}^{2}+2 \sum_{k=1}^{n-2} k F_{k}^{2}+\sum_{k=0}^{n-2} F_{k}^{2} \\
& \geq F_{2 n-1} F_{2 n-2}+F_{2 n-2}^{2}-3 F_{n-1} F_{n-2}-2 F_{n-2}^{2}+F_{n} F_{n-1}+\left[1-(-1)^{n}\right] \\
& \|A\|_{2} \geq \sqrt{\frac{F_{2 n-1} F_{2 n-2}+F_{2 n-2}^{2}-3 F_{n-1} F_{n-2}-2 F_{n-2}^{2}+F_{n} F_{n-1}+\left[1-(-1)^{n}\right]}{n}} .
\end{aligned}
$$

On the other hand, let the matrices $B$ and $C$ be $n \times n$ matrices satisfying 
$A=B \circ C$.

$$
B=\left[\begin{array}{cccc}
r & r & \cdots & 1 \\
r & & \cdots & 1 \\
\vdots & \vdots & \ddots & \vdots \\
1 & 1 & \cdots & 1
\end{array}\right] \text { and } C=\left[\begin{array}{cccc}
F_{0} & F_{1} & \cdots & F_{n-1} \\
F_{1} & & \cdots & F_{n} \\
\vdots & \vdots & \ddots & \vdots \\
F_{n-1} & F_{n} & \cdots & F_{2 n-2}
\end{array}\right]
$$

Since

$$
r_{1}(B)=\max _{i} \sqrt{\sum_{j}\left|b_{i j}\right|^{2}}=\sqrt{\sum_{j=0}^{n-1}\left|b_{n j}\right|^{2}}=\sqrt{|r|^{2}(n-1)+1}
$$

and

$$
c_{1}(C)=\max _{j} \sqrt{\sum_{i}\left|c_{i j}\right|^{2}}=\sqrt{\sum_{i=0}^{n-1}\left|c_{i n}\right|^{2}}=\sqrt{\sum_{i=n-1}^{2 n-2} F_{i}^{2}}=\sqrt{F_{2 n-1} F_{2 n-2}-F_{n-1} F_{n-2}},
$$

we have

$$
\|A\|_{2} \leq \sqrt{\left(|r|^{2}(n-1)+1\right)\left(F_{2 n-1} F_{2 n-2}-F_{n-1} F_{n-2}\right)} .
$$

when $|r|<1$ we also obtain

$$
\begin{gathered}
A_{F}^{2} \geq|r|^{2}\left[\sum_{k=0}^{n-2}(k+1) F_{k}^{2}+\sum_{k=n-1}^{2 n-2}(2 n-1-k) F_{k}^{2}\right] \\
\geq|r|^{2}\left[(2 n-1) \sum_{k=0}^{n-2} F_{k}^{2}-\sum_{k=1}^{2 n-2} k F_{k}^{2}-(2 n-1) \sum_{k=0}^{n-2} F_{k}^{2}+2 \sum_{k=1}^{n-2} k F_{k}^{2}+\sum_{k=0}^{n-2} F_{k}^{2}\right] \\
\geq|r|^{2}\left[F_{2 n-1} F_{2 n-2}+F_{2 n-2}^{2}-3 F_{n-1} F_{n-2}-2 F_{n-2}^{2}+F_{n} F_{n-1}+\left[1-(-1)^{n}\right]\right] \\
\|A\|_{2} \geq|r| \sqrt{\frac{F_{2 n-1} F_{2 n-2}+F_{2 n-2}^{2}-3 F_{n-1} F_{n-2}-2 F_{n-2}^{2}+F_{n} F_{n-1}+\left[1-(-1)^{n}\right]}{n}} .
\end{gathered}
$$

On the other hand, let the matrices $B$ and $C$ be $n \times n$ matrices satisfying $A=B \circ C$.

$$
B=\left[\begin{array}{cccc}
r & r & \cdots & 1 \\
r & & \cdots & 1 \\
\vdots & \vdots & \ddots & \vdots \\
1 & 1 & \cdots & 1
\end{array}\right] \text { and } C=\left[\begin{array}{cccc}
F_{0} & F_{1} & \cdots & F_{n-1} \\
F_{1} & & \cdots & F_{n} \\
\vdots & \vdots & \ddots & \vdots \\
F_{n-1} & F_{n} & \cdots & F_{2 n-2}
\end{array}\right]
$$

Since

$$
r_{1}(B)=\max _{i} \sqrt{\sum_{j}\left|b_{i j}\right|^{2}}=\sqrt{\sum_{j=0}^{n-1}\left|b_{n j}\right|^{2}}=\sqrt{n}
$$

and

$$
c_{1}(C)=\max _{j} \sqrt{\sum_{i}\left|c_{i j}\right|^{2}}=\sqrt{\sum_{i=0}^{n-1}\left|c_{i n}\right|^{2}}=\sqrt{\sum_{i=n-1}^{2 n-2} F_{i}^{2}}=\sqrt{F_{2 n-1} F_{2 n-2}-F_{n-1} F_{n-2}},
$$

we have

$$
\|A\|_{2} \leq \sqrt{n\left(F_{2 n-1} F_{2 n-2}-F_{n-1} F_{n-2}\right)} .
$$

Thus, the proof is concluded.

Theorem 2.7: Let $A=H_{r}=\left(a_{i j}\right)$ be a Hankel matrix satisfying $a_{i j}=L_{i+j-2}$, where $r \in(\mathcal{C})$. 
1) $|r| \geq 1,\|A\|_{2} \geq \begin{cases}\sqrt{\frac{L_{2 n-1} L_{2 n-2}-2 L_{n-1} L_{n-2}+L_{2 n-2}^{2}-2 L_{n-2}^{2}+1}{n}}, & \text { if } n \text { is odd } \\ \sqrt{\frac{L_{2 n-1} L_{2 n-2}-2 L_{n-1} L_{n-2}+L_{2 n-2}^{2}-2 L_{n-2}^{2}+6}{n}}, & \text { otherwise }\end{cases}$

$$
\|A\|_{2} \leq \sqrt{\left(|r|^{2}(n-1)+1\right)\left(L_{2 n-1} L_{2 n-2}-L_{n-1} L_{n-2}\right)}
$$

2) $|r|<1,\|A\|_{2} \geq\left\{\begin{array}{l}|r| \sqrt{\frac{L_{2 n-1} L_{2 n-2}-2 L_{n-1} L_{n-2}+L_{2 n-2}^{2}-2 L_{n-2}^{2}+1}{n},}, \text { if } n \text { is odd } \\ |r| \sqrt{\frac{L_{2 n-1} L_{2 n-2}-2 L_{n-1} L_{n-2}+L_{2 n-2}^{2}-2 L_{n-2}^{2}+6}{n}}, \text { otherwise }\end{array}\right.$

$$
\|A\|_{2} \leq \sqrt{n\left(L_{2 n-1} L_{2 n-2}-L_{n-1} L_{n-2}\right)}
$$

where $L_{n}$ is the spectral norm and $L_{n}$ denotes the $n$th Lucas number.

Proof. The matrix $A$ is of the form

$$
A=\left[\begin{array}{cccc}
r F_{0} & r F_{1} & \cdots & F_{n-1} \\
r F_{1} & & \cdots & F_{n} \\
\vdots & \vdots & \ddots & \vdots \\
F_{n-1} & F_{n} & \cdots & F_{2 n-2}
\end{array}\right]
$$

Then,

$$
\|A\|_{F}^{2}=\sum_{k=0}^{n-2}|r|^{2}(k+1) L_{k}^{2}+\sum_{k=n-1}^{2 n-2}(2 n-1-k) L_{k}^{2} .
$$

Hence, when $|r| \geq 1$ we obtain

$$
\begin{aligned}
& A_{F}^{2} \geq \sum_{k=0}^{n-2}(k+1) L_{k}^{2}+\sum_{k=n-1}^{2 n-2}(2 n-1-k) L_{k}^{2} \\
& \geq(2 n-1) \sum_{k=0}^{n-2} L_{k}^{2}-\sum_{k=n-1}^{2 n-2} k L_{k}^{2}-(2 n-1) \sum_{k=0}^{n-2} L_{k}^{2}+2 \sum_{k=1}^{n-2} k L_{k}^{2}+\sum_{k=0}^{n-2} L_{k}^{2} \\
& \geq\left\{\begin{array}{l}
L_{2 n-1} L_{2 n-2}-2 L_{n-1} L_{n-2}+L_{2 n-2}^{2}-2 L_{n-2}^{2}+1, \text { if } n \text { is odd } \\
L_{2 n-1} L_{2 n-2}-2 L_{n-1} L_{n-2}+L_{2 n-2}^{2}-2 L_{n-2}^{2}+6, \text { otherwise }
\end{array}\right. \\
&\|A\|_{2} \geq\left\{\begin{array}{l}
\sqrt{\frac{L_{2 n-1} L_{2 n-2}-2 L_{n-1} L_{n-2}+L_{2 n-2}^{2}-2 L_{n-2}^{2}+1}{n},}, \text { if } n \text { is odd } \\
\sqrt{\frac{L_{2 n-1} L_{2 n-2}-2 L_{n-1} L_{n-2}+L_{2 n-2}^{2}-2 L_{n-2}^{2}+6}{n}}, \text { otherwise }
\end{array}\right.
\end{aligned}
$$

On the other hand, let the matrices $B$ and $C$ be $n \times n$ matrices satisfying $A=B \circ C$.

$$
B=\left[\begin{array}{cccc}
r & r & \cdots & 1 \\
r & & \cdots & 1 \\
\vdots & \vdots & \ddots & \vdots \\
1 & 1 & \cdots & 1
\end{array}\right] \text { and } C=\left[\begin{array}{cccc}
L_{0} & L_{1} & \cdots & L_{n-1} \\
L_{1} & & \cdots & L_{n} \\
\vdots & \vdots & \ddots & \vdots \\
L_{n-1} & L_{n} & \cdots & L_{2 n-2}
\end{array}\right]
$$

Since

$$
r_{1}(B)=\max _{i} \sqrt{\sum_{j}\left|b_{i j}\right|^{2}}=\sqrt{\sum_{j=0}^{n-1}\left|b_{n j}\right|^{2}}=\sqrt{|r|^{2}(n-1)+1}
$$

and 


$$
c_{1}(C)=\max _{j} \sqrt{\sum_{i}\left|c_{i j}\right|^{2}}=\sqrt{\sum_{i=0}^{n-1}\left|c_{i n}\right|^{2}}=\sqrt{\sum_{i=n-1}^{2 n-2} L_{i}^{2}}=\sqrt{L_{2 n-1} L_{2 n-2}-L_{n-1} L_{n-2}},
$$

we have

$$
\|A\|_{2} \leq \sqrt{\left(|r|^{2}(n-1)+1\right)\left(L_{2 n-1} L_{2 n-2}-L_{n-1} L_{n-2}\right)} .
$$

when $|r|<1$ we also obtain

$$
\begin{aligned}
\|A\|_{F}^{2} & \geq|r|^{2}\left[\sum_{k=0}^{n-2}(k+1) L_{k}^{2}+\sum_{k=n-1}^{2 n-2}(2 n-1-k) L_{k}^{2}\right] \\
& \geq|r|^{2}\left[(2 n-1) \sum_{k=0}^{n-2} L_{k}^{2}-\sum_{k=1}^{2 n-2} k L_{k}^{2}-(2 n-1) \sum_{k=0}^{n-2} L_{k}^{2}+2 \sum_{k=1}^{n-2} k L_{k}^{2}+\sum_{k=0}^{n-2} L_{k}^{2}\right] \\
& \geq|r|^{2}\left\{\begin{array}{l}
L_{2 n-1} L_{2 n-2}-2 L_{n-1} L_{n-2}+L_{2 n-2}^{2}-2 L_{n-2}^{2}+1, \text { if } n \text { is odd } \\
L_{2 n-1} L_{2 n-2}-2 L_{n-1} L_{n-2}+L_{2 n-2}^{2}-2 L_{n-2}^{2}+6, \text { otherwise }
\end{array}\right. \\
& \|A\|_{2} \geq\left\{\begin{array}{l}
|r| \sqrt{\frac{L_{2 n-1} L_{2 n-2}-2 L_{n-1} L_{n-2}+L_{2 n-2}^{2}-2 L_{n-2}^{2}+1}{n}}, \text { if } n \text { is odd } \\
|r| \sqrt{\frac{L_{2 n-1} L_{2 n-2}-2 L_{n-1} L_{n-2}+L_{2 n-2}^{2}-2 L_{n-2}^{2}+6}{n}}, \text { otherwise }
\end{array}\right.
\end{aligned}
$$

On the other hand, let the matrices $B$ and $C$ be $n \times n$ matrices satisfying $A=B \circ C$.

$$
B=\left[\begin{array}{cccc}
r & r & \cdots & 1 \\
r & & \cdots & 1 \\
\vdots & \vdots & \ddots & \vdots \\
1 & 1 & \cdots & 1
\end{array}\right] \text { and } C=\left[\begin{array}{cccc}
L_{0} & L_{1} & \cdots & L_{n-1} \\
L_{1} & & \cdots & L_{n} \\
\vdots & \vdots & \ddots & \vdots \\
L_{n-1} & L_{n} & \cdots & L_{2 n-2}
\end{array}\right]
$$

Since

$$
r_{1}(B)=\max _{i} \sqrt{\sum_{j}\left|b_{i j}\right|^{2}}=\sqrt{\sum_{j=0}^{n-1}\left|b_{n j}\right|^{2}}=\sqrt{n}
$$

and

$$
c_{1}(C)=\max _{j} \sqrt{\sum_{i}\left|c_{i j}\right|^{2}}=\sqrt{\sum_{i=0}^{n-1}\left|c_{i n}\right|^{2}}=\sqrt{\sum_{i=n-1}^{2 n-2} L_{i}^{2}}=\sqrt{L_{2 n-1} L_{2 n-2}-L_{n-1} L_{n-2}},,
$$

we have $\|A\|_{2} \leq \sqrt{n\left(L_{2 n-1} L_{2 n-2}-L_{n-1} L_{n-2}\right)}$.

Thus, the proof is concluded.

\section{Numerical Examples}

Example 3.8: Let $A=H_{r}\left(F_{0}, F_{1}, \cdots, F_{n-1}\right)$ be a $r$-Hankel matrix where $r \in(\mathcal{C})$. It can easily be seen that the values obtained in theorem 2.6. are more closure than those obtained in lemma 1.1. to exact values in from Table 2.

Example 3.9: Let $A=H_{r}\left(L_{0}, L_{1}, \cdots, L_{n-1}\right)$ be a $r$-Hankel matrix where $r \in(C)$. It can easily be seen that the values obtained in theorem 2.7. are more closure than those obtained in lemma 1.3. to exact values in from Table 3.

Table 2. Numerical results of $a_{i j}=F_{i+j-2}, r=1$.

n Lemma 1.1. Theorem 2.6. $\quad$ Lemma 1.2. (exact value)




\section{Continued}

\begin{tabular}{cccc}
\hline 2 & $\sqrt{4}$ & $\sqrt{4}$ & $\frac{1+\sqrt{5}}{2}$ \\
3 & $\sqrt{84}$ & $\sqrt{42}$ & $\frac{4+\sqrt{28}}{2}$ \\
4 & $\sqrt{3978}$ & $\sqrt{408}$ & $\frac{12+\sqrt{180}}{2}$ \\
5 & $\sqrt{189744}$ & $\sqrt{3540}$ & $\frac{33+\sqrt{1185}}{2}$ \\
6 & $\sqrt{9057280}$ & $\sqrt{29280}$ & $\frac{88+\sqrt{7674}}{2}$ \\
$\vdots$ & $\vdots$ & $\vdots$ & $\vdots$ \\
\hline
\end{tabular}

Table 3. Numerical results of $a_{i j}=L_{i+j-2}, r=1$.

\begin{tabular}{cccc}
\hline $\mathrm{n}$ & Lemma 1.3. & Theorem 2.7. & Lemma 1.4. (exact value) \\
\hline 2 & $\sqrt{20}$ & $\sqrt{20}$ & $\frac{5+\sqrt{5}}{2}$ \\
3 & $\sqrt{1184}$ & $\sqrt{222}$ & $\frac{12+\sqrt{84}}{2}$ \\
4 & $\sqrt{95370}$ & $\sqrt{2040}$ & $\frac{30+\sqrt{720}}{2}$ \\
5 & $\sqrt{4708068}$ & $\sqrt{17720}$ & $\frac{77+\sqrt{5449}}{2}$ \\
6 & $\sqrt{226236800}$ & $\sqrt{146400}$ & $\frac{200+\sqrt{38720}}{2}$ \\
$\vdots$ & $\vdots$ & $\vdots$ & $\vdots$ \\
\hline
\end{tabular}

\section{Conclusion}

In this paper, we firstly define the $r$-Hankel matrix with entries Fibonacci and Lucas numbers. Then we introduce the Euclidean norm equality and the spectral norm inequalities of these matrices. Furthermore, we compared our finding with the exact value of matrix $A$ 's spectral norm. In the future we shall further develop some kinds of $r$-Hankel matrix such as right $r$-Hankel, left $r$-Hankel and geometric $r$-Hankel. Also we will observe upper and lower bounds for the spectral norm of these matrices involving famous number sequences.

\section{References}

[1] Solak, S. (2005) On the Norms of Circulant Matrices with the Fibonacci and Lucas Numbers. Applied Mathematics and Computation, No. 160, 125-132. https://doi.org/10.1016/j.amc.2003.08.126

[2] Türkmen, R. and Gökbaş, H. (2016) On the Soectral Norm of $r$-Circulant Matrices with Pell and Pell-Lucas Numbers. Journal of Inequalities and Applications, 2016, 65. https://doi.org/10.1186/s13660-016-0997-0

[3] Shen, S. and Cen, J. (2010) On the Spectral Norms of $r$-Circulant Matrices with the 
k-Fibonacci and k-Lucas Numbers. International Journal of Contemporary Mathematical Sciences, 5, 569-578.

[4] Akbulak, M. and Bozkurt, D. (2008) On the Norms of Toeplitz Matrices Involving Fibonacci and Lucas Numbers. Hacettepe Journal of Mathematics and Statistics, 37 89-95.

[5] Shen, S. (2012) On the Norms of Toeplitz Matrices Ivolving $k$-Fibonacci and $k$-Lucas Numbers. International Journal of Contemporary Mathematical Sciences, 7, 363-368.

[6] Akbulak, M. and Bozkurt, D. (2008) On the Norms of Hankel Matrices Involving Fibonacci and Lucas Numbers. Selçuk Journal of Applied Mathematics, 9, 45-52.

[7] Gökbaş, H. and Türkmen, R. (2016) On the Norms of $r$-Toeplitz Matrices Involving Fibonacci and Lucas Numbers, Advances in Linear Algebra Matrix Theory, No. 6, 31-39.

[8] Bozkurt, D. and Tam, T. (2012) Determinants and Inverse of Circulant Matrices with Jacobsthal and Jacobsthal-Lucas Numbers. Applied Mathematics and Computation, 2, 544-551. https://doi.org/10.1016/j.amc.2012.06.039

[9] Koshy, T. (2001) Fibonacci and Lucas Numbers with Applications. A Wiley-Interscience Publication, Canada.

[10] Horn, R.A. and Johnson, C.R. (1991) Topics in Matrix Analysis. Cambridge University Press, New York. https://doi.org/10.1017/CBO9780511840371

[11] Solak, S. and Bahşi, M. (2011) On the Spectral Norms of Hankel Matrices with Fibonacci and Lucas Numbers. Selçuk Journal of Applied Mathematics, 12, 71-76. 